\title{
The combination of microRNA-371a-3p and 375-5p can distinguish viable germ cell tumor and teratoma from necrosis in postchemotherapy retroperitoneal lymph node dissection specimens
}

\author{
Lara Kremer ${ }^{1}$, Melanie von Brandenstein ${ }^{1}$, Maike Wittersheim ${ }^{2}$, Barbara Koeditz ${ }^{1}$, Pia Paffenholz ${ }^{1}$, \\ Martin Hellmich ${ }^{3}$, David Pfister ${ }^{1}$, Axel Heidenreich ${ }^{1}$, Tim Nestler ${ }^{1,4} \wedge$ \\ ${ }^{1}$ Department of Urology, University of Cologne, Faculty of Medicine and University Hospital Cologne, Cologne, Germany; ${ }^{2}$ Institute of Pathology, \\ University of Cologne, Faculty of Medicine and University Hospital Cologne, Cologne, Germany; ${ }^{3}$ Institute of Medical Statistics and Computational \\ Biology, University of Cologne, Faculty of Medicine and University Hospital Cologne, Cologne, Germany; ${ }^{4}$ Department of Urology, Federal Armed \\ Services Hospital Koblenz, Koblenz, Germany \\ Contributions: (I) Conception and design: L Kremer, T Nestler; (II) Administrative support: A Heidenreich, T Nestler; (III) Provision of study \\ materials and patients: M Wittersheim, P Paffenholz, D Pfister, A Heidenreich; (IV) Collection and assembly of data: L Kremer, M von Brandenstein, \\ B Koeditz; (V) Data analysis and interpretation: L Kremer, M von Brandenstein, M Hellmich, T Nestler; (VI) Manuscript writing: All authors; (VII) \\ Final approval of manuscript: All authors. \\ Correspondence to: Dr. med. Tim Nestler. Department of Urology, Uro-Oncology, Robot Assisted and Reconstructive Urologic Surgery University \\ Hospital Cologne, Kerpener Straße 62, 50937 Cologne, Germany. Email: tim.nestler@uk-koeln.de.
}

Background: To identify a combination of microRNAs (miRNA) to differentiate between viable tumor (V) or teratoma $(\mathrm{T})$ and necrosis/fibrosis $(\mathrm{N})$ in pcRPLND specimens of metastatic germ cell tumor (GCT) patients with residual masses $\geq 1 \mathrm{~cm}$ after chemotherapy. Biomarker guided therapy could reduce overtreatment with pcRPLND in patients with only $\mathrm{N}$.

Methods: We selected 48 metastatic GCT patients who had undergone pcRPLND. V, pure $\mathrm{T}$ and $\mathrm{N}$ was shown in the resected tissue of 16 patients, respectively. Of these areas total RNA was isolated and miRNA expression was analyzed for miR-371a-3p, 375-3p, and 375-5p using qPCR. ROC analysis was performed for each miRNA and for all combinations in order to determine the discriminatory capacity of $\mathrm{V}$ and $\mathrm{T} v s$. N.

Results: On comparing V vs. N miR-371a-3p achieved the highest fold change (FC) of $31.1(\mathrm{P}=0.023)$ while for $\mathrm{T}$ vs. $\mathrm{N}$ miR-375-5p performed best (FC 64.2; $\mathrm{P}<0.001$ ). Likewise, the most accurate AUC for $\mathrm{V}$ was 0.75 using miR-371a-3p, for T 0.80 using miR-375-5p. Combining the best performing miRNAs for $\mathrm{V}$ and $\mathrm{T}$ resulted in an AUC of 0.94 with a sensitivity of 93.75 , specificity of 93.75 , PPV of 96.8 and NPV of 83.3 .

Conclusions: By combining miR-371a-3p and miR-375-5p in pcRPLND tissue samples $V$ and $T$ could be distinguished from necrosis/fibrosis with great accuracy. This combination of miRNAs might serve as new biomarker in the future, in order to spare miRNA-negative patients from pcRPLND. However, further studies analyzing patient's serum are needed to confirm the clinical impact of these biomarkers.

Keywords: miR-371-3p; miR-375; post-chemotherapy lymph node dissection; testicular cancer; germ cell tumor (GCT)

Submitted Oct 19, 2020. Accepted for publication Jan 22, 2021.

doi: $10.21037 /$ tau-20-1349

View this article at: http://dx.doi.org/10.21037/tau-20-1349

^ ORCID-ID: 0000-0001-6033-6364. 


\section{Introduction}

Metastatic non-seminomatous testicular germ cell tumors (GCT) with remaining metastatic tissue of $\geq 1 \mathrm{~cm}$ on CT scan after chemotherapy should be completely resected during postchemotherapy retroperitoneal lymph node dissection (pcRPLND) $(1,2)$. However, histopathological examinations reveal only necrosis/fibrosis in $50 \%$ of all patients, which are thus relevantly overtreated. To optimize the presurgical diagnostic accuracy different attempts have been made. Various clinical prediction models were proposed using tumor marker decline, shrinkage of retroperitoneal masses, presence of teratoma and other parameters. However, accuracy of these models was not appropriate enough for routine use (3-5). Also new image processing methods, such as radiomics, have already shown good results in a single series, but it is not sufficient for decision-making yet (6). Within the last few years, miR-371a-3p has been proposed as a new and highly accurate biomarker for GCT (7-10). A recent study has investigated miR-371a-3p in serum of patients prior to pcRPLND to discriminate between viable GCT or teratoma and necrosis, resulting in a promising area under the curve (AUC) of 0.874 (11). However, the main limitation of miR-371a-3p is its negativity for teratoma $(7,8,11)$, hence there is need for another miRNA that, in combination with miR-371a-3p could close this "teratoma gap”. A large study that characterized GCT regarding its molecular level using different sequencing methods, proposed miR-375 as a potential biomarker for teratoma (12). Few groups have additionally investigated miR-375 in serum so far (13-15). Until now, further investigations of the miRNA subtypes miR-375-3p and miR-375-5p are scarce. Therefore, the aim of this study was to investigate whether a miRNA of miR-375 is differentially expressed in teratoma tissue compared so necrosis/fibrosis. If so, it should be tested if a combination of miR-371a-3p with one of these mature miRNAs could discriminate viable GCT or teratoma from necrosis/fibrosis on tissue level.

We present the following article in accordance with the STARD reporting checklist (available at http://dx.doi. org/10.21037/tau-20-1349).

\section{Methods}

\section{Patients and samples}

STARD guidelines were followed to perform this study (16). The study was conducted in accordance with the Declaration of Helsinki (as revised in 2013). The study was approved by the local ethics committee of University Hospital of Cologne (18-280) and informed consent was taken from all the patients. Metastatic non-seminomatous GCT patients were selected retrospectively for the study and were identified using the clinical database of the Department of Urology at the University Hospital of Cologne. All patients had given written consent and underwent orchiectomy, chemotherapy and pcRPLND. pcRPLND was performed for retroperitoneal residual tumors $\geq 1 \mathrm{~cm}$ in patients with normal or stable tumor markers at the time of completion of chemotherapy. All histological slides were reviewed by an experienced uropathologist (MW), classified according to 2016 World Health Organization recommendations (17) and staged according to the TNM classification 7 th edition (18). Risk classification was determined according to the International Germ Cell Consensus Classification (IGCCCG) for metastatic, malignant TGCTs (19). Histology of pcRPLND tumors was classified as necrosis/ fibrosis, pure teratoma or viable GCT (non-seminoma or seminoma). In cases with a mixed histology, patients with viable GCT and concomitant teratoma were classified as viable GCT, while a combination of teratoma and necrosis was classified as teratoma. Representative areas of metastatic tumors were identified and selectively collected for subsequent RNA extraction.

\section{miRNA purification and quantitative real-time PCR (qRT-PCR)}

Macrodissection of target areas was performed using multiple $10 \mu \mathrm{m}$ thick formalin-fixed paraffin-embedded (FFPE) tissue sections. Total RNA was extracted using miRNeasy FFPE Kit (Qiagen®, Hilden, Germany), according to manufacturer's instructions. RNA samples were quantified using QuantiFluor ${ }^{\circledR}$ (Promega, Madison, WI, USA). For qRT-PCR $100 \mathrm{ng}$ were required.

For the analysis of the miRNAs miScriptR II RT (Qiagen ${ }^{\circledR}$, Hilden, Germany) and SYBR ${ }^{\circledR}$ Green PCR Master mix (Applied Biosystems ${ }^{\mathrm{TM}}$, Foster City, CA, USA) was used according to the manufacture's protocol. qRT-PCR was carried out in 96-well plates using Quantstudio ${ }^{\mathrm{TM}} 7$ Flex RealTime PCR (Applied Biosystems ${ }^{\mathrm{TM}}$, Foster City, CA, USA), according to the recommended protocol, starting at $95^{\circ} \mathrm{C}$ for $20 \mathrm{~s}$, followed by 40 cycles at $95^{\circ} \mathrm{C}$ for $15 \mathrm{~s}, 50^{\circ} \mathrm{C}$ for $30 \mathrm{~s}$ and $72{ }^{\circ} \mathrm{C}$ for $30 \mathrm{~s}$ and terminated at $72{ }^{\circ} \mathrm{C}$ for $15 \mathrm{~s}$.

For the analysis of miRNAs the following primers were used: hsa-miR-371a-3p, GUGCCGCCAUCUUUUGAGUGU; hsa-miR-375-3p, UUUGUUCGUUCGGCUCGCGUGA; 
hsa-miR-375-5p, GCGACGAGCCCUCGCACAAACC; 5s rRNA, GGCCAUACCACCCUGAACGC.

5s rRNA (MystiCq ${ }^{\circledR}$ Universal PCR Primer, SigmaAldrich, St. Louis, MO, USA) was used for quantification, RNA of tumor-free tissue as a positive control. All experiments were done in triplicates. miRNA levels were determined according to the $\Delta \Delta \mathrm{C}_{\mathrm{T}}$ method.

\section{Statistical analysis}

Data were analyzed by descriptive statistics. Pearson correlation was used to test the association of miRNAs with different variable. Intergroup differences were compared using ANOVA. Differences in miRNA expression levels among histological subtypes were assessed using KruskalWallis test and tests adjusted to ties. Bonferroni correction was performed for multiple comparisons. We utilized receiver operating characteristic (ROC) curves to display the predictive ability of different miRNA signatures graphically, followed by calculating AUC (95\% CI), and optimal cut-point determination using the Youden-Index method. According to optimal cut-points biomarker performance parameters (sensitivity, specificity, positive predictive value (PPV), negative predictive value (NPV) and accuracy) were calculated. To calculate ROC curves with the combination of miRNAs we used bivariate logistic regression. A two-tailed $\mathrm{P}$ value of $<0.05$ was considered statistically significant. Microsoft Excel for Mac and IBM SPSS statistics V26.0 was used for analysis.

\section{Results}

Our study cohort consisted of 48 metastatic GCT patients and was split in three groups (each with $\mathrm{n}=16$ patients) representing (I) only viable GCT, (II) pure teratoma and (III) necrosis/fibrosis in the pcRPLND specimens. Patients' characteristics are shown in Table 1. Patients with pure teratoma were younger compared to patients with viable GCT and necrosis/fibrosis (mean age 29.0, 33.8, 38.3 years, $\mathrm{P}=0.031$ ). Levels of common tumor marker of GCT [alpha fetoprotein (AFP), hCG, LDH] prior to pcRPLND were normal in all patients except for two with elevated but stable AFP (558 and 2,013 kU/L) and two with elevated but stable hCG (24 and $23 \mathrm{U} / \mathrm{L})$. Histopathological examination of pcRPLND specimens showed teratoma in the patients with elevated AFP. Viable GCT and teratoma was present in the two patients with elevated hCG. Tissue levels of miR-371a$3 p$, miR-375-3p and miR-375-5p showed no significant correlation with the above-mentioned serum tumor markers prior to pcRPLND.

In a next step, we evaluated the ability of miRNAs to discriminate between viable GCT, pure teratoma and necrosis/fibrosis. Patients harboring viable GCT had significantly elevated levels of miR-371a-3p $(\mathrm{P}=0.023)$, miR$375-3 \mathrm{p}(\mathrm{P}=0.016)$ and miR-375-5p $(\mathrm{P}=0.004)$ compared to patients exhibiting only necrosis. Levels of miR-371a$3 \mathrm{p}$ were significantly higher in viable GCT compared to teratoma $(\mathrm{P}=0.009)$. In teratoma, levels of miR-375-3p and miR-375-5p were significantly elevated compared to necrosis $(\mathrm{P}=0.001, \mathrm{P}<0.001$; Figure 1$)$.

To determine the discriminative value of the miRNAs regarding pathohistological findings in the pcRPLND specimens we performed ROC analysis. miR-371a$3 \mathrm{p}$ was best in predicting viable GCT with an AUC of 0.752, compared to 0.572 for miR-375-3p and miR-375$5 \mathrm{p}$, respectively (Figure S1). Teratoma was determined most accurately by miR-375-5p (AUC of 0.797), followed by miR-375-3p (AUC 0.729) and miR-371a-3p (AUC 0.348) (Figure S2). Regarding the discrimination of both pathohistological findings, viable GCT and teratoma, miR$375-5 \mathrm{p}$ performed best with an AUC of 0.869 , followed by miR-375-3p (AUC 0.801) and miR-371a-3p (0.600) (Figure 2). The corresponding statistical quality criteria of diagnostic tests such as sensitivity, specificity, PPV and NPV are shown in Table 2. Subsequently, we tested every combination of the three miRNAs. The combination of miR-371a-3p and miR-375-5p achieved the best AUC of 0.938, AUC for miR-371a-3p and miR-375-3p was 0.861 and for miR-375-3p and miR-375-5p was 0.912 (Figure 2). Only the combination of all three miRNAs achieved an even slightly better AUC of 0.96 . The best combination of two miRNAs (371a-3p and 375-5p) had a sensitivity of 93.8 , specificity of 93.8 , PPV of 96.8 and NPV of 83.2 (Table 2). Here, only one patient was misclassified in each group: viable GCT (embryonal carcinoma less than 5\% of the resected specimen), teratoma (less than $5 \%$ in the whole residual tissue) and necrosis/fibrosis, respectively (corresponding contingency tables are shown in Figure S3).

The calculated AUCs for serum tumor markers AFP, hCG and LDH evaluated after chemotherapy to determine viable GCT and teratoma in our cohort of patients were $0.613,0.428$, and 0.559 , respectively (Figure S4).

\section{Discussion}

As $50 \%$ of all patients treated by pcRPLND due to residual 
Table 1 Patient characteristics and clinicopathological data

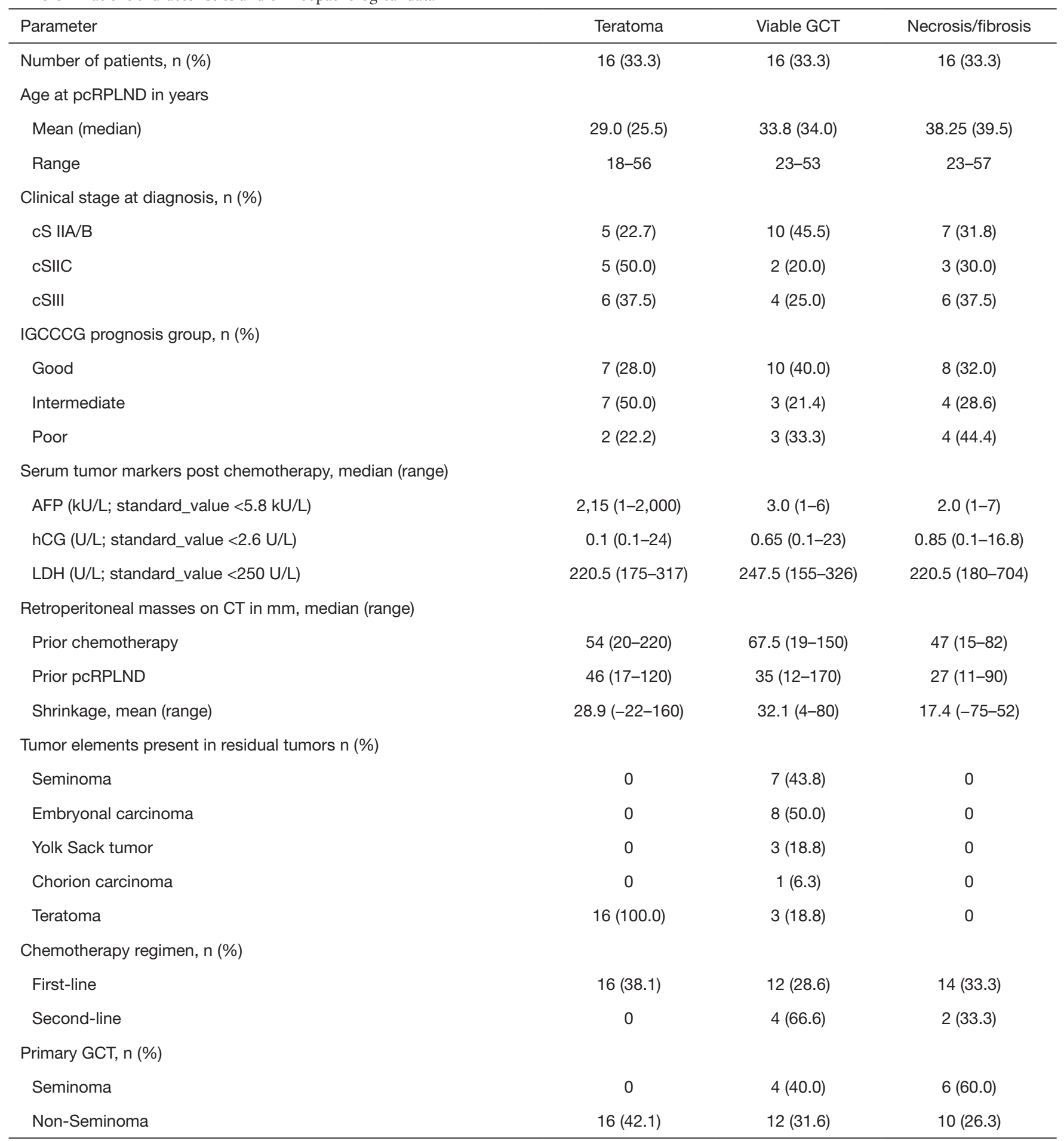

GCT, germ cell tumor; pcRPLND, postchemotherapy retroperitoneal lymph node dissection; cS, Clinical Stage; IGCCCG, International Germ Cell Cancer Collaborative Group; AFP, $\alpha$-fetoprotein; hCG, human chorionic gonadotropin; LDH, lactate dehydrogenase; CT, computed tomography. 
A

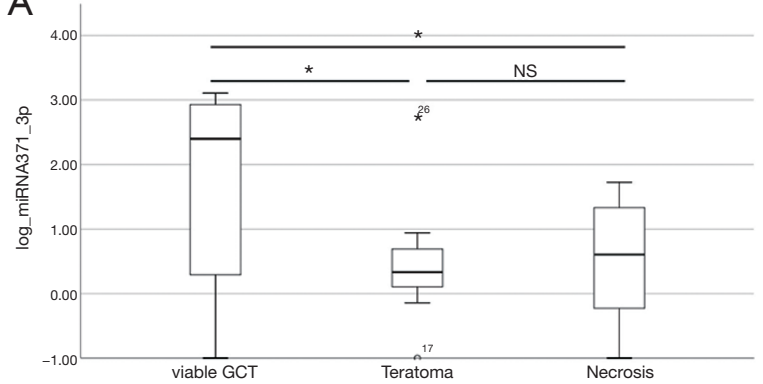

B

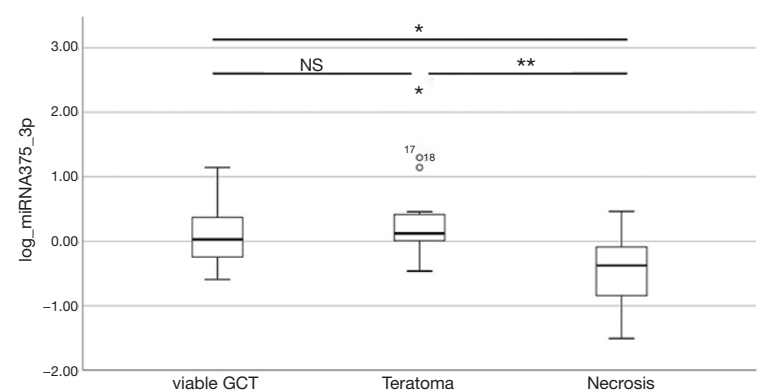

C

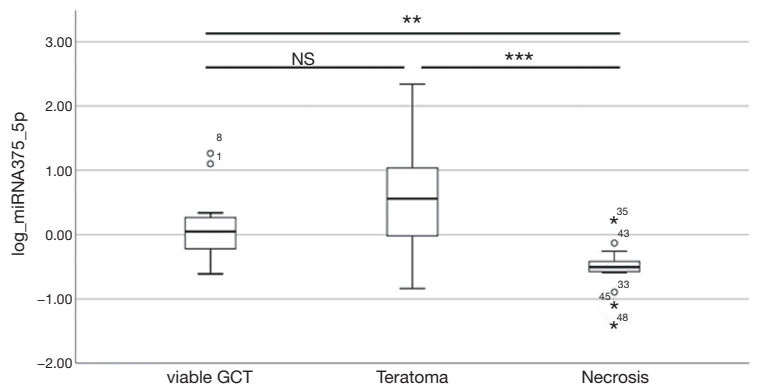

Figure 1 MiRNA levels in pcRPLND specimen harboring viable germ cell tumor (GCT), teratoma and necrosis. (A) MiR-371a-3p, (B) miR-375-3p, (C) miR-375-5p showing significant different expression levels between viable GCT and necrosis ( $\mathrm{A}$ : * $\mathrm{P}=0.023$; $\mathrm{B}$ : * $\mathrm{P}=0.016$; $\left.\mathrm{C}:{ }^{*} \mathrm{P}=0.004\right)$. B. and $\mathrm{C}$. have significant different expression levels between teratoma and necrosis $\left(\mathrm{B}:{ }^{* *} \mathrm{P}=0.001 ; \mathrm{C}:{ }^{* * *} \mathrm{P}<0.001\right)$ while levels between viable GCT and teratoma are similar except for miR-371a-3p ( $\mathrm{P}=0.009)$. NS, non-significant.

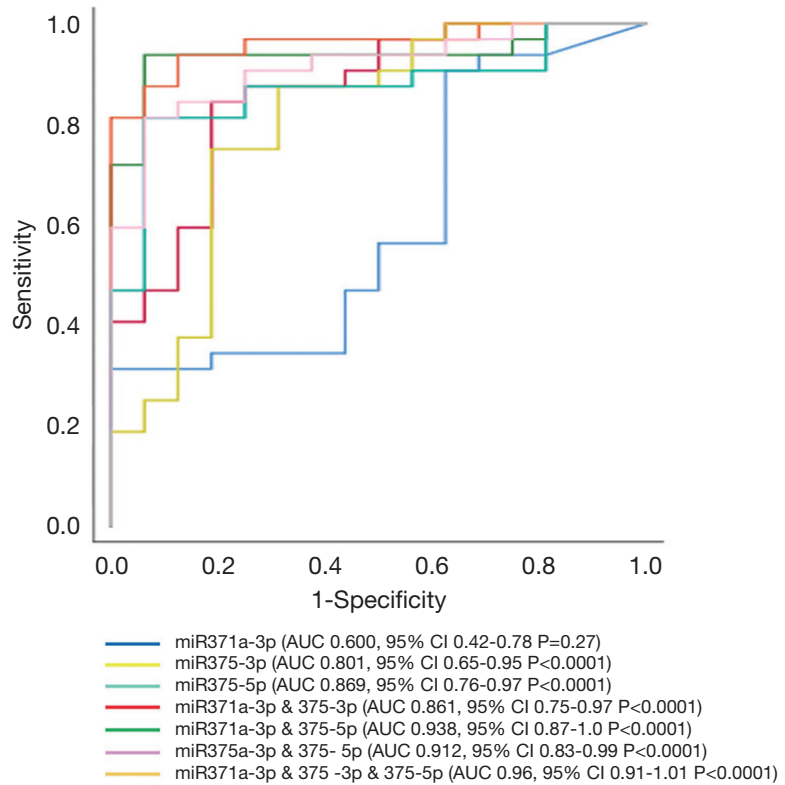

Figure 2 ROC curves of all miRNAs and all combinations with each other to predict viable GCT and teratoma. The best combination is miR-371a-3p and miR-375-5p. ROC, receiver operating characteristic; GCT, germ cell tumor. metastasis after chemotherapy harbor only necrosis/fibrosis in pcRPLND specimens, the objective of this study was to reduce the overtreatment in this group of patients. So far, only miR-371a-3p has been proposed as a new biomarker to predict viable GCT prior pcRPLND with promising results. As teratoma cannot be predicted using this miRNA, we evaluated the use of miR375 regarding the prediction of pathohistological finding in pcRPLND. Furthermore, we explored three miRNAs with regard to their ability to determine viable GCT and teratoma in contrast to necrosis/ fibrosis in pcRPLND specimens.

In our study, levels of miR-371a-3p were significantly higher in residual tissue with viable GCT compared to those with pure teratoma or necrosis/fibrosis. This is in line with the current knowledge of miR-371a-3p, as various studies have shown a high sensitivity and specificity for this miRNA to differentiate between viable GCT and teratoma or benign histology $(7,8,11,15,20)$. Of note, the sensitivity of miR-371a-3p was much lower in our study compared to the previously mentioned studies. Since most previous studies have only been performed on serum prior to pcRPLND and not on tissue of the residual metastasis, 
Table 2 Calculation of sensitivity, specificity, PPV, NPV according to optimal cut-points of ROC curves for miR-371a-3p, miR-375-3p, miR-375-5p, and miR-371a-3p + miR-375-5p to predict viable GCT, teratoma or both

\begin{tabular}{|c|c|c|c|c|c|}
\hline & Specificity & Sensitivity & PPV & NPV & AUC \\
\hline V vs. N & 100 & 56.3 & 93.8 & 69.6 & 0.752 \\
\hline T vs. N & 100 & 6.3 & 93.8 & 51.6 & 0.348 \\
\hline VT vs. N & 100 & 31.3 & 96.8 & 42.1 & 0.6 \\
\hline V vs. N & 81.3 & 62.5 & 76.9 & 68.4 & 0.572 \\
\hline T vs. N & 81.3 & 87.5 & 82.4 & 86.7 & 0.729 \\
\hline VT vs. N & 81.3 & 75.0 & 88.9 & 61.9 & 0.801 \\
\hline \multicolumn{6}{|l|}{$\operatorname{miR}-375-5 p$} \\
\hline VT vs. N & 93.8 & 81.3 & 96.3 & 71.4 & 0,869 \\
\hline \multicolumn{6}{|c|}{ miR-371a-3p + miR-375-5p } \\
\hline V vs. N & 93.8 & 93.8 & 93.8 & 93.8 & - \\
\hline T vs. N & 93.8 & 93.8 & 93.8 & 93.8 & - \\
\hline VT vs. N & 93.8 & 93.8 & 96.8 & 88.2 & 0.938 \\
\hline
\end{tabular}

Cut-off values were: miR-371a-3p >103.4, miR-375-3p >1.0, miR-375-5p >0.76. Corresponding contingency tables Table S1. V, viable germ cell tumor; N, necrosis; T, teratoma; PPV, positive predictive value; NPV, negative predictive value; AUC, area under the curve.

it might be possible that chemotherapy-induced reduction of miR-371a-3p expression in the remaining viable GCT may occur, while the release of miR-371a-3p into serum is largely maintained. Investigation on GCT tissue are scarce. Murray et al. analyzed pediatric GCT tissue and found miRNA cluster 371-373 to be overexpressed in GCT (21). Shen $e t a l$. did a comprehensive investigation of molecular characteristics on primary GCT tissue of adults and found miR-371a-3p to be overexpressed in GCT subtypes except for teratoma (12). Again, the main differences to our patients were pediatric $v s$. adults in the first study and primary orchiectomy specimen $v s$. pcRPLND tissue in both last-mentioned studies.

Since miR-371a-3p cannot sufficiently detect teratoma we were aiming for an additional miRNA to close this "teratoma gap". Recently, Shen et al. described miR375 to be highly expressed in tissue of teratomas, yolk sac tumors, and mixed tumors containing these elements, but not in seminoma or embryonal carcinoma (12). As prior studies have only described miR-375 in general, we analyzed both subtypes miR-375-3p and miR-375-5p in
pcRPLND specimens for the first time. Both subtypes showed significantly elevated levels in teratoma compared to necrosis/fibrosis with even higher levels for miR-375-5p compared to miR-375-3p $(\mathrm{P}>0.001$ vs. $\mathrm{P}=0.001)$. Likewise, expression levels of both miRNAs were significantly elevated in viable GCT compared to necrosis, too. Again, miR-375$5 \mathrm{p}$ was able to distinguish more accurately between viable GCT and necrosis than miR-375-3p ( $\mathrm{P}=0.004$ vs. $\mathrm{P}=0.016)$. However, there were no significant different expression levels between teratoma and viable GCT. In conclusion, these different expression levels support the hypothesis that miR-375 and in particular its subtypes miR-375-3p/miR$375-5 \mathrm{p}$ could be beneficial to distinguish teratoma or viable GCT and necrosis.

Recently, miR-375 has been studied by some groups with contradicting results to predict teratoma (13-15,20). In contrast to our study, all mentioned studies evaluated miRNAs in serum and not in tissue samples, Lobo et al. investigated different miRNAs to differentiate teratoma from healthy controls (14). They could not confirm miR375 to be upregulated in serum of teratoma bearing 
patients. However, the miRNA subtypes miR-375-3p and miR-375-5p were not included in their miRNA-array. Lafin et al. showed that miR-375-3p was not informative for the detection of teratoma, too (20). They investigated serum of patients prior to primary retroperitoneal lymph node dissection. However, it must be noted that their cohort of patients only included three patients with teratoma in retroperitoneal histology. Nappi and coworkers were the first who did an integrated expression analysis of circulating miR-371a-3p and miR-375 to identify teratoma and viable malignant GCT components (15). In their discovery cohort miR375 expression was significantly higher in patients with residual teratoma than in patients with CSI $(\mathrm{P}=0.01)$ and $\mathrm{AUC}$ of miR375 was 0.93 . miR371a-3p was only expressed in metastatic seminoma but not in teratoma or clinical stage I patients on surveillance $(\mathrm{P}<0.0001)$. The AUC of combined miR371 and miR375 was 0.95 . Their validation cohort consisted of 38 patients prior to pcRPLND with teratoma in 21 cases, 6 with active GCT, and 11 with complete response. The AUC of combined miR375-miR371 was 0.77 and higher than that of individual miR371 (AUC 0.74) and miR375 (AUC 0.55). Their results emphasize the impact of different timepoints in GCT therapy for establishing cut points for miRNA diagnostics. Interestingly, the discovery cohort performed much better for combined miR375-miR371 compared to the validation cohort with pcRPLND patients. As the combination of both miRNAs was better than the individual miRNAs in the discovery and in the validation cohort, we should further on focus on a miRNA panel to differentiate viable GCT/teratoma from necrosis. Belge and colleagues exclusively analyzed the miR-375-3p subtype but not miR$375-5 p$ in serum of GCT patients before orchiectomy (13). The authors did not find miR-375-3p to be sufficient to differentiate between teratoma/viable GCT and healthy controls (AUC 0.524). According to our data we assume that especially subtype miR-375-5p might be slightly better to predict teratoma. Therefore, we would suggest to further investigate both miRNAs (miR-375-3p/-5p) in prospective studies.

Currently the data on miR-375 for accurate teratoma detection remains unclear. Consequently, further studies are warrant to examine the predictive capacity of the miRNA375 subtypes in combination with miRNA371a-3p in larger cohorts of GCT patients.

Next, we studied the ability of the three miRNAs and their combinations to discriminate teratoma or viable GCT from necrosis. To discriminate teratoma and necrosis
miR-375-5p had the best AUC (0.797), for viable GCT $v s$. necrosis miR-371a-3p was best with an AUC of 0.752 . The AUC for miR-371a-3p was lower compared to other studies which performed serum analysis for GCT, showing AUCs of up to $0.966(7,8,20,22-24)$. This could be due to different timepoints during treatment, as the highest AUCs were described prior to chemotherapy. The only study that investigated miR-371a-3p on serum of patients prior to pcRPLND reported an AUC of 0.874 for viable GCT, which was thus lower in comparison to those levels prior to treatment $(7,8,11)$. In this study by Leao et al., expression levels between patients harboring teratoma and necrosis did not show significant differences. Another explanation for the different AUC in this setting might be due to the analyzed material, the aforementioned studies tested miR-371a-3p in serum while we analyzed tissue samples. The difference in miR-371a-3p expression might also be explained by different patient characteristics and clinical settings.

As we hypothesized that a combination of two miRNAs would best discriminate viable GCT and teratoma from necrosis, we tested all combinations of the studied miRNAs. The best results were achieved for the combination of miR-371a-3p and miR-375-5p, resulting in an AUC of 0.938 (95\% CI: $0.87-1.0, \mathrm{P}<0.0001)$ and a sensitivity and specificity of $93.75 \%$, respectively. This combination of miRNAs led to a correct discrimination of 45 of all 48 tissue samples, using the thresholds based on the optimal cutpoints of the ROC. Two samples out of 32 with viable GCT or teratoma were misdiagnosed as necrosis, and one sample out of 16 with necrosis/fibrosis was misclassified as viable GCT or teratoma. Of the misclassified tissues one contained teratoma with less than $5 \%$ in the whole residual tissue, the other viable embryonal carcinoma also with less than $5 \%$ of the whole resected specimen. As those missed tumors were very small, viable areas might have been missed at macrodissection. Additionally, in the largest study on serum levels of miR-371a-3p in testis cancer patients, it was shown that tumor sizes $<1 \mathrm{~cm}$ showed inferior sensitivity in serum analysis (8). From that data it appears rational to assume that small areas of residual viable GCT or teratoma within a large residual mass might also be missed by serum testing of the combined test miR-371a-3p/miR-375-5. Obviously, a minimum number of vital tumor cells is required to produce measurable serum levels.

The major limitation of our study was its retrospective design. Although our cohort of studied patients was small, we selected patients carefully for balanced groups of final 
histopathology (viable GCT, teratoma and necrosis, each with $\mathrm{n}=16$ patients) including more patients with viable GCT in pcRPLND compared to the studied cohorts of Leao et al. (11) and the pcRPLND cohort of Nappi et al. (15). Same group sizes should lead to a better statistical comparability of the studied miRNAs. Another relevant limitation is the lack of external validation of our findings on patient's serum, which, however, corresponds to the discovery character of our study.

\section{Conclusions}

The combination of miR-371a-3p and miR-375-5p showed excellent results in the distinction of viable GCT/ teratoma and necrosis/fibrosis in pcRPLND tissue. As a result, the combination of these two miRNAs might spare pcRPLND in miRNA-negative patients in order to reduce overtreatment. However, further studies analyzing patient's serum are needed to confirm the clinical impact of these biomarkers.

\section{Acknowledgments}

Funding: This work was supported by Southwest German Society for Urology (Südwestdeutsche Gesellschaft für Urologie, SWDGU).

\section{Footnote}

Reporting Checklist: The authors have completed the STARD reporting checklist. Available at http://dx.doi.org/10.21037/ tau-20-1349

Data Sharing Statement: Available at http://dx.doi. org/10.21037/tau-20-1349

Conflicts of Interest: All authors have completed the ICMJE uniform disclosure form (available at http://dx.doi. org/10.21037/tau-20-1349). LK reports grants from Koeln Fortune Program/Faculty of Medicine, University of Cologne, during the conduct of the study. Dr. TN reports grants from Research Grant of Southwest German Society for Urology, during the conduct of the study. The other authors have no conflicts of interest to declare.

Ethical Statement: The authors are accountable for all aspects of the work in ensuring that questions related to the accuracy or integrity of any part of the work are appropriately investigated and resolved. The study was conducted in accordance with the Declaration of Helsinki (as revised in 2013). The study was approved by the local ethics committee of University Hospital of Cologne (18-280) and informed consent was taken from all the patients.

Open Access Statement: This is an Open Access article distributed in accordance with the Creative Commons Attribution-NonCommercial-NoDerivs 4.0 International License (CC BY-NC-ND 4.0), which permits the noncommercial replication and distribution of the article with the strict proviso that no changes or edits are made and the original work is properly cited (including links to both the formal publication through the relevant DOI and the license). See: https://creativecommons.org/licenses/by-nc-nd/4.0/.

\section{References}

1. Albers $\mathrm{P}$, Albrecht $\mathrm{W}$, Algaba F, et al. Guidelines on Testicular Cancer: 2015 Update. Eur Urol 2015;68:1054-68.

2. Honecker F, Aparicio J, Berney D, et al. ESMO Consensus Conference on testicular germ cell cancer: diagnosis, treatment and follow-up. Ann Oncol 2018;29:1658-86.

3. Paffenholz P, Nestler T, Hoier S, et al. Independent validation of two models to predict necrosis/fibrosis in post chemotherapy residual retroperitoneal masses of patients with advanced testicular cancer Eur Urol Suppl 2019;18:827.

4. Leão R, Nayan M, Punjani N, et al. A New Model to Predict Benign Histology in Residual Retroperitoneal Masses After Chemotherapy in Nonseminoma. Eur Urol Focus 2018;4:995-1001.

5. Steyerberg EW, Keizer HJ, Sleijfer DT, et al. Retroperitoneal metastases in testicular cancer: role of CT measurements of residual masses in decision making for resection after chemotherapy. Radiology 2000;215:437-44.

6. Baessler B, Nestler T, Pinto Dos Santos D, et al. Radiomics allows for detection of benign and malignant histopathology in patients with metastatic testicular germ cell tumors prior to post-chemotherapy retroperitoneal lymph node dissection. Eur Radiol 2020;30:2334-45.

7. Dieckmann KP, Radtke A, Spiekermann M, et al. Serum Levels of MicroRNA miR-371a-3p: A Sensitive and Specific New Biomarker for Germ Cell Tumours. Eur Urol 2017;71:213-20.

8. Dieckmann KP, Radtke A, Geczi L, et al. Serum Levels of MicroRNA-371a-3p (M371 Test) as a New Biomarker of Testicular Germ Cell Tumors: Results of a Prospective 
Multicentric Study. J Clin Oncol 2019;37:1412-23.

9. Nappi L, Thi M, Lum A, et al. Developing a Highly Specific Biomarker for Germ Cell Malignancies: Plasma miR371 Expression Across the Germ Cell Malignancy Spectrum. J Clin Oncol 2019;37:3090-8.

10. Mego M, van Agthoven T, Gronesova P, et al. Clinical utility of plasma miR-371a-3p in germ cell tumors. J Cell Mol Med 2019;23:1128-36.

11. Leão R, van Agthoven T, Figueiredo $A$, et al. Serum miRNA Predicts Viable Disease after Chemotherapy in Patients with Testicular Nonseminoma Germ Cell Tumor. J Urol 2018;200:126-35.

12. Shen H, Shih J, Hollern DP, et al. Integrated Molecular Characterization of Testicular Germ Cell Tumors. Cell Rep 2018;23:3392-406.

13. Belge G, Grobelny F, Matthies C, et al. Serum Level of microRNA-375-3p Is Not a Reliable Biomarker of Teratoma. In Vivo 2020;34:163-8.

14. Lobo J, Gillis AJM, van den Berg A, et al. Identification and Validation Model for Informative Liquid BiopsyBased microRNA Biomarkers: Insights from Germ Cell Tumor In Vitro, In Vivo and Patient-Derived Data. Cells 2019;8:1637.

15. Nappi L, Thi M, Adra N, et al. Integrated Expression of Circulating miR375 and miR371 to Identify Teratoma and Active Germ Cell Malignancy Components in Malignant Germ Cell Tumors. Eur Urol 2021;79:16-9.

16. Cohen JF, Korevaar DA, Altman DG, et al. STARD 2015 guidelines for reporting diagnostic accuracy studies: explanation and elaboration. BMJ Open 2016;6:e012799.

17. Moch H, Cubilla AL, Humphrey PA, et al. The 2016

Cite this article as: Kremer $\mathrm{L}$, von Brandenstein $M$, Wittersheim M, Koeditz B, Paffenholz P, Hellmich M, Pfister D, Heidenreich A, Nestler T. The combination of microRNA$371 a-3 p$ and $375-5 p$ can distinguish viable germ cell tumor and teratoma from necrosis in postchemotherapy retroperitoneal lymph node dissection specimens. Transl Androl Urol 2021;10(4):1647-1655. doi: 10.21037/tau-20-1349
WHO Classification of Tumours of the Urinary System and Male Genital Organs-Part A: Renal, Penile, and Testicular Tumours. Eur Urol 2016;70:93-105.

18. Edge SB, Compton CC. The American Joint Committee on Cancer: the 7th edition of the AJCC cancer staging manual and the future of TNM. Ann Surg Oncol 2010;17:1471-4.

19. International Germ Cell Consensus Classification: a prognostic factor-based staging system for metastatic germ cell cancers. International Germ Cell Cancer Collaborative Group. J Clin Oncol 1997;15:594-603.

20. Lafin JT, Singla N, Woldu SL, et al. Serum MicroRNA371a-3p Levels Predict Viable Germ Cell Tumor in Chemotherapy-naive Patients Undergoing Retroperitoneal Lymph Node Dissection. Eur Urol 2020;77:290-2.

21. Murray MJ, Saini HK, van Dongen S, et al. The two most common histological subtypes of malignant germ cell tumour are distinguished by global microRNA profiles, associated with differential transcription factor expression. Mol Cancer 2010;9:290.

22. Gillis AJ, Stoop HJ, Hersmus R, et al. High-throughput microRNAome analysis in human germ cell tumours. J Pathol 2007;213:319-28.

23. Murray MJ, Halsall DJ, Hook CE, et al. Identification of microRNAs From the miR-371 373 and miR-302 clusters as potential serum biomarkers of malignant germ cell tumors. Am J Clin Pathol 2011;135:119-25.

24. Syring I, Bartels J, Holdenrieder S, et al. Circulating serum miRNA (miR-367-3p, miR-371a-3p, miR-372-3p and $\mathrm{miR}-373-3 \mathrm{p}$ ) as biomarkers in patients with testicular germ cell cancer. J Urol 2015;193:331-7. 


\section{Supplementary}

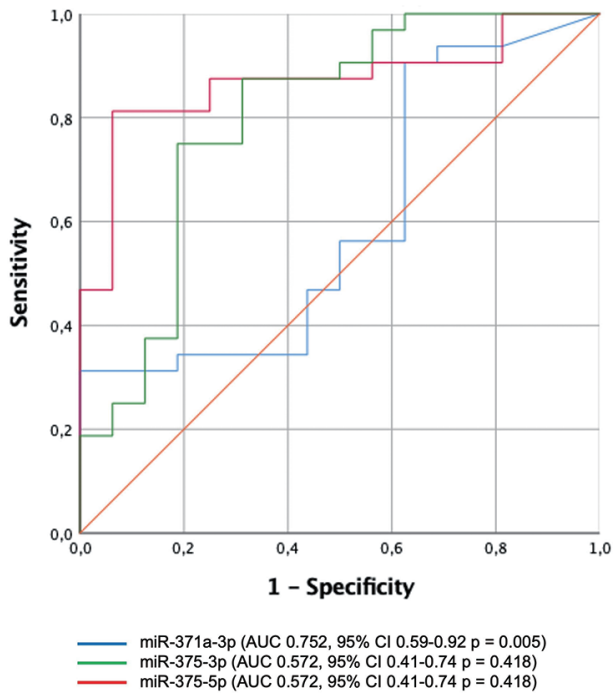

Figure S1 ROC curves for the three miRNAs to predict viable GCT. GCT, germ cell tumor.

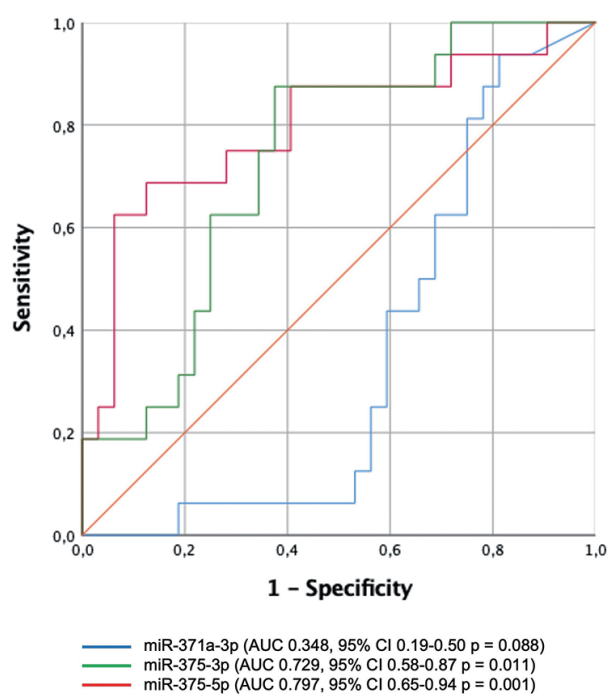

Figure S2 ROC curves for the three miRNAs to predict teratoma.
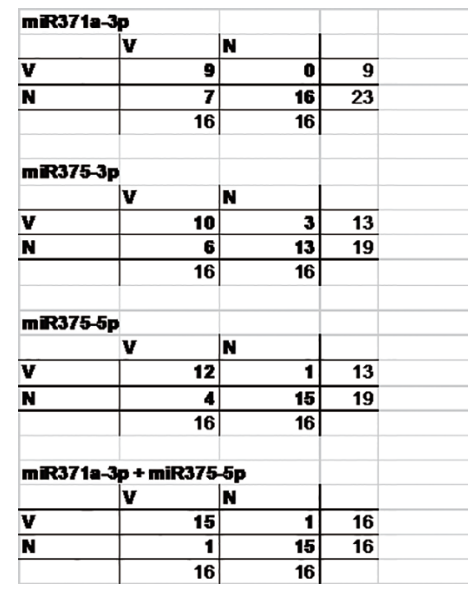

Figure S3 Contingency table for miR-371a-3p, miR-375-3p, miR371-5p, and miR-371a-3p + miR375-5p to predict viable GCT, Teratoma or both according to optimal cut-points of ROC curves. Cut-off values were: miR-371a-3p >103.4, mirR375-3p > 1.0, miR-375-5p >0.76. 


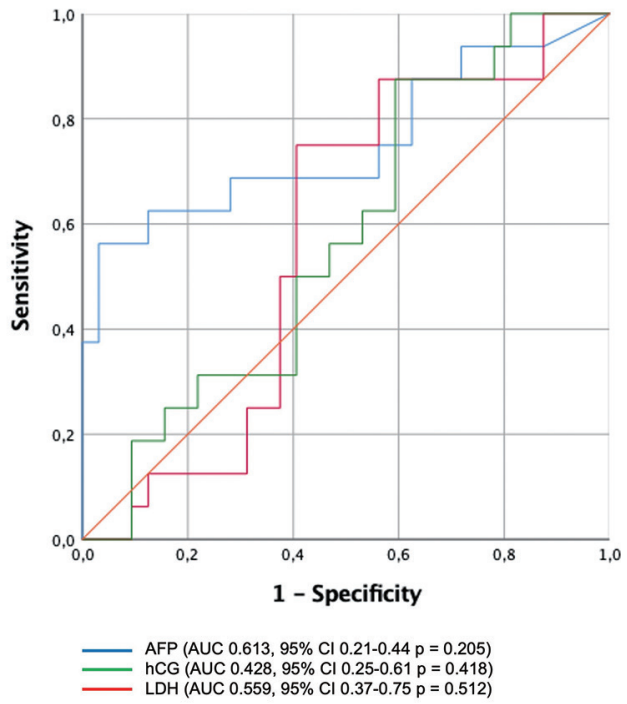

Figure S4 ROC curves for the serum tumor markers AFP, hCG and LDH to predict viable GCT and Teratoma. GCT, germ cell tumor; AFP, $\alpha$-fetoprotein, hCG, human chorionic gonadotropin; LDH, lactate dehydrogenase. 